\title{
Variable Structure Control for Hypersonic Vehicle Based on Model Reference
}

\author{
Wei Jianli1 ${ }^{*}$, TianShida ${ }^{1}$, Yao Xinghua ${ }^{2}$ \\ ${ }^{1}$ College of Astronautics, Northwestern Polytechnical University, 127 West Youyi Road, Xi'an Shaanxi, \\ 710072, P. R. China. \\ 2 Ming De College, Northwestern Polytechnical University, Chang'an Xi'an Shaanxi, 710124, P. R. China. \\ * Corresponding author. Tel.:+86 029-88492331; email: weijianli@mail.nwpu.edu.cn \\ Manuscript submitted November 4, 2014; accepted January 17, 2015. \\ doi: 10.17706/ijapm.2015.5.2.144-152
}

\begin{abstract}
A hypersonic vehicle has a large flight envelope span, complicated flight environment, glaring external perturbation and unknown factor interference, being difficult to establish its precise model. Furthermore, the advanced airframe-engine integrated design technique makes it sensitive to change in flight conditions, thus challenging the design of its control system. We design the model reference variable structural control (MRVSC) scheme that can resist the internal structural parametric variation and external perturbation of the control system. The scheme has the merits of both adaptive control and variable structural control, can effectively track the reference model and resist the great parametric variation, effectively enhancing the robustness of the control system of the hypersonic vehicle. We design its pitch channel controller and roll channel controller with the model reference variable structural control theory, evaluate the performances of main design points of each channel and perform the 6-DOF simulation to verify the control system. The verification results show preliminarily that the step responses of the MRVSC we designed are basically unchangeable in the two states that the hypersonic vehicle has its cowling or no cowling, while the PID controller cannot ensure that the step responses are unchangeable when the state changes, indicating that the MRVSC scheme is superior to the PID controller when the control has uncertainties. The 6-DOF simulation results further verify that the MRVSC scheme can better resist great parametric variation and has strong robustness.
\end{abstract}

Key words: Classic PID, hypersonic vehicle, model reference variable structure control, robustness.

\section{Introduction}

A hypersonic vehicle refers to a type of militarily important flight vehicle whose Mach number exceeds 5 . Different from an ordinary low-speed aircraft, the hypersonic vehicle has a large flight envelope span and a complicated flight environment. The advanced airframe-propulsion system integrated design technique causes the mutual interference between the propulsion system and the control rudder surface; and the aerodynamic coefficients are complicated functions between angle of attack and the Mach number. Meanwhile, the hypersonic vehicle flies under highly dynamic pressure with glaring external interference and unknown factor interference, causing the complexity and uncertainty of its aerodynamic characteristics [1]. Therefore, it is difficult to establish its precise model and design its control system.

Due to the above unknown factors and uncertainties of the hypersonic vehicle, variable structural control and adaptive control are widely applied to its effective and robust control. To solve the strong coupling, 
severe nonlinearity and variable coefficients of the hypersonic vehicle during its re-entry stage, reference [2] proposed an adaptive and robust variable structural control algorithm that can overcome the shortcomings of traditional control algorithms. Reference [3] designed a terminal sliding mode variable structural control scheme by introducing the concept of ultimate attractor in neural networks, enabling the tracking error to converge to the balance point zero within a limited time. Based on the application of the SISO uncertain linear system, Yu et al proposed a rapid terminal sliding mode variable structural control scheme in [4] and [5]. Reference [6] designed a variable structure controller based on Lyapunov stabilization theory and Riccati equation of the generalized correlational large-scale system. It makes the sliding-mode satisfy the reachability condition, and the correlational large-scale system is global structural asymptotic stability. Reference [7] introduced an adaptive parameter adjuster control rule. This method can effectively improve the performance of dynamics and stable characteristics in the pitch channel, and it also has strong adaptability to the changes of aerodynamic parameters and elevator trimming.

Based on the above research, we propose the MRVSC scheme that can resist great parametric variation. This method combines the advantages of model reference adaptive and variable structure control algorithm, and can rapidly track the reference model and resist the great parametric variation, effectively enhancing the robustness of the control system of the hypersonic vehicle.

\section{Mathematical Model of a Hypersonic Vehicle}

For an axial symmetry aircraft with STT control scheme, coupling of pitch channel, yaw channel and roll channel is small and can be ignored, and kinetic characteristic of yaw channel and roll channel are basically the same and control system of pitch, yaw and roll channel can be designed independently. Mathematical model of longitudinal flight control of a hypersonic vehicle can be seen in [8] and [9].

Dynamic equations of lateral flight control of a hypersonic vehicle are

$$
\left\{\begin{array}{l}
\dot{\omega}_{x}=\frac{M_{x}\left(V, \omega_{x}, \delta_{x}\right)}{J_{x}} \\
\dot{\gamma}=\omega_{x}
\end{array}\right.
$$

where $V$ is velocity, $\gamma$ is roll angle, $\omega_{x}$ is roll rate. $\delta_{x}, J_{z}$, and $M_{x}$ represent aileron deflection, moment of inertia about roll axis and rolling moment respectively.

\section{Design of MRVSC [10], [11]}

\subsection{Description of Mathematical Model}

\subsubsection{Mathematical description of controlled plant}

Mathematical model of general uncertainty multivariable system is shown as follow:

$$
X_{p}=\left(A_{p}+\Delta A_{p}\right) X_{p}+\left(B_{p}+\Delta B_{p}\right) u+D_{p} f_{p}
$$

where $X \in R^{n}$ is state variable, $u \in R^{m}$ is control variable, $f_{p} \in R^{l}$ is external interference; $A_{p} \in R^{n \times n}$, and $B_{p} \in R^{n \times m}$ are nominal matrix and nominal control matrix of controlled plant respectively; $\Delta A_{p} \in R^{n \times n}$, $\Delta B_{p} \in R^{n \times m}$, and $D_{p} \in R^{n \times l}$ are perturbation matrices of $A_{p}, B_{p}$ and perturbation distribution matrix.

\subsubsection{Mathematical description of reference model}

Mathematical model of general reference model is described as follow: 


$$
X_{m}=A_{m} X_{m}+B_{m} u_{m}
$$

\subsubsection{Mathematical model of error}

Mathematical model of error is defined as follow:

$$
e(t)=X_{m}(t)-X_{p}(t)
$$

which can be further described as:

$$
e=A_{m} e(t)+\left[A_{m}-A_{p}\right] x_{p}+B_{p} u(t)-\Delta A_{p} x_{p}-\Delta B_{p} u(t)-D_{p} f_{p}(t)
$$

In order to simplify control system design, using the elementary row transformation matrix $T$, $z=T e$, the transformation form of error model is equal to:

$$
z=\tilde{A}_{m} z(t)+A_{m p} x(t)+\tilde{B}_{m} u_{m}-\tilde{B} u(t)-\Delta \tilde{A}_{p} x_{p}-\Delta \tilde{B}_{p} u(t)-\tilde{D} f(t)
$$

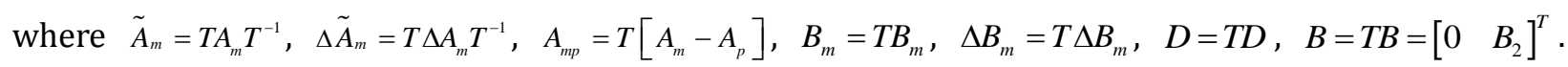

\subsection{Choice of Sliding Hyperplane}

In allusion to the error model, choosing the following sliding hyperplane:

$$
S=C e
$$

where the sliding mode matrix $C$ should be greater than zero, $C=\left[\begin{array}{ll}C_{1} & C_{2}\end{array}\right] \in R^{m \times n}$ is a full rank matrix, and $C_{1} \in R^{m \times(n-m)}, C_{2} \in R^{m \times m}$.

\subsection{Choice of Control Law}

The form of MRVSC controller is written as $u=u_{m r}+u_{v s}$ usually, where $u_{m r}$ is matching control law of closed-loop control system of model reference, and $u_{v s}$ is variable structure control law.

\subsubsection{Choice of reference model control law}

In order to achieve complete tracking, there must be $\lim _{t \rightarrow \infty} e=0$, which means there is a proper $u$ to ensure the following equation having solution.

$$
A_{m p} x+B_{m} u_{m}-B u=0
$$

And the following equation is obtained:

$$
u_{m r}=B_{2}^{-1}\left[\begin{array}{ll}
0 & I_{m}
\end{array}\right] A_{m p} x_{p}+B_{2}^{-1}\left[\begin{array}{ll}
0 & I_{m}
\end{array}\right] B_{m} r
$$

\subsubsection{Choice of variable structure control law}

The selected variable structure controller $u_{v s}$ shall ensure to keep the system on the sliding mode stable and reliable, that is to say there is a right $u_{v s}$ to guarantee $V=S^{T} S<0$, if $u_{v s}=g(t)\left(C B_{p}\right)^{-1} \operatorname{sgn}(S), V$ 
can be written as:

$$
\begin{aligned}
V= & -g(t) S^{T} \operatorname{sgn}(S)-g(t) S^{T} C \Delta B_{p}\left(C B_{p}\right)^{-1} \operatorname{sgn}(S)-S^{\mathrm{T}} C \Delta B_{p} u_{m}+S^{T}\left[C A_{m} z-C \Delta A_{p} x_{p}-C D_{p} f_{p}\right] \leq \\
& -g(t)\left\|S^{T}\right\|\left[1-\left\|\Delta B_{p}\right\|\|C\|\left\|\left(C B_{p}\right)^{-1}\right\|\right]+\left\|S^{T}\right\|\left\|\Delta B_{p}\right\|\|C\|\left\|u_{m}\right\|+\left\|S^{T}\right\|\left[\left\|C A_{m}\right\|\|z\|\right. \\
& \left.-\left\|\Delta A_{p}\right\|\|C\|\left\|x_{p}\right\|-\left\|C D_{p}\right\|\left\|f_{p}\right\|\right]
\end{aligned}
$$

where

$$
g(t)=\left(1-p_{5}\right)^{-1}\left[p_{1}\|z\|+p_{2}\left\|x_{p}\right\|+p_{3}\left\|u_{m}\right\|+p_{4}\right]+\varepsilon
$$

It can make sure that $V<0$ and keep the system on the sliding mode stable and reliable. Where $\varepsilon$ is a small positive number, $\quad p_{1}=\left\|C A_{m}\right\|, \quad p_{2}=\left\|\Delta A_{p}\right\|\|C\|, \quad p_{3}=\left\|\Delta B_{p}\right\|\|C\|, \quad p_{4}=\left\|f_{p}\right\|\left\|C D_{p}\right\|$, $p_{5}=\left\|\Delta B_{p}\right\|\|C\|\left\|\left(C B_{p}\right)^{-1}\right\|$, and $\|\bullet\|$ means induced norm.

In order to eliminate the high frequency flutter, substitute the following equation $m_{i}(s)$ for $\operatorname{sgn}\left(s_{i}\right)$.

$$
m_{i}(s)=\frac{s_{i}}{\left|s_{i}\right|+\delta_{i}}
$$

In summary, the design steps of MRVSC are: obtain the reference model which meets the model matching condition of entirely tracking through model reference planning methods firstly, then design a sliding mode matrix $C$ based on the error system, and finally obtain the variable structure tracking control law to ensure the controlled plant tracks the reference model effectively.

\section{MRVSC Design and Simulation of Pitch Channel}

\subsection{MRVSC Design of Pitch Channel}

The detail design procedures are shown in part 3 and no more explanation is presented here. State equation of reference model can be described as:

$$
\left[\begin{array}{c}
\dot{n}_{y m} \\
\dot{\omega}_{z m}
\end{array}\right]=\left[\begin{array}{cc}
0 & \frac{V \bar{a}_{4}}{g} \\
g & -\frac{2 \xi_{m}}{T_{m}}
\end{array}\right]\left[\begin{array}{l}
n_{y m} \\
\omega_{z m}
\end{array}\right]+\left[\begin{array}{c}
0 \\
\frac{g}{T_{m}^{2} V \bar{a}_{4}}
\end{array}\right] n_{y c}
$$

The reference model control law $u_{m r}$ can be written as follow:

$$
u_{m r}=\left(\frac{g}{a_{3} a_{4} V T_{m}^{2}}-\frac{g a_{2}}{a_{3} a_{4} V}\right) n_{y}-\frac{g}{a_{3} a_{4} V T_{m}^{2}} n_{y c}+\left(\frac{-2 \xi_{m}}{T_{m}}+a_{1}\right) \omega_{z}
$$

And the variable structure control law $u_{v s}$ is shown in the following equation:

$$
u_{v s}=\frac{g(t)}{-a_{3}} \operatorname{sgn}(S)
$$

\subsection{Performance Check of the Main Design Point of Pitch Channel}


State vector of pitch channel is $X=\left[\begin{array}{ll}n_{y} & \omega_{z}\end{array}\right]^{T}$, and the schematics of control system is shown as Fig. 1.

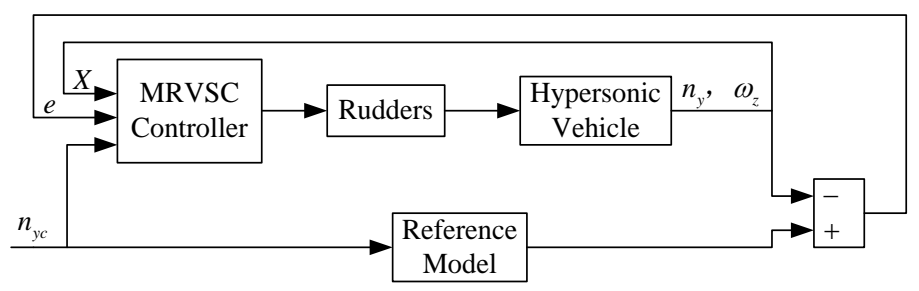

Fig. 1. Control system of pitch channel.

Keep the control parameters unchanged, and compare the control effect of MRVSC and PID under two main design point though digital simulation.

Main design point 1: With cowling, velocity is $5.5 \mathrm{Ma}$, altitude is $24000 \mathrm{~m}$ and angle of attack is $2^{\circ}$.

Main design point 2: Without cowling, velocity is $6.0 \mathrm{Ma}$, altitude is $26000 \mathrm{~m}$ and angle of attack is $2^{\circ}$.

Ignore the influence of rate gyro and actuator. Time-domain characteristics of main design point are shown in Fig. 2 and Fig. 3.

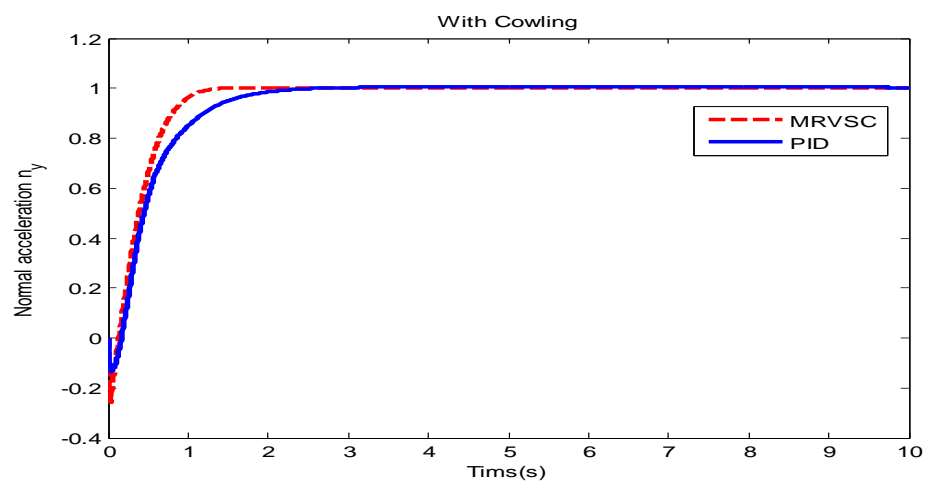

Fig. 2. Normal acceleration step response with cowling.

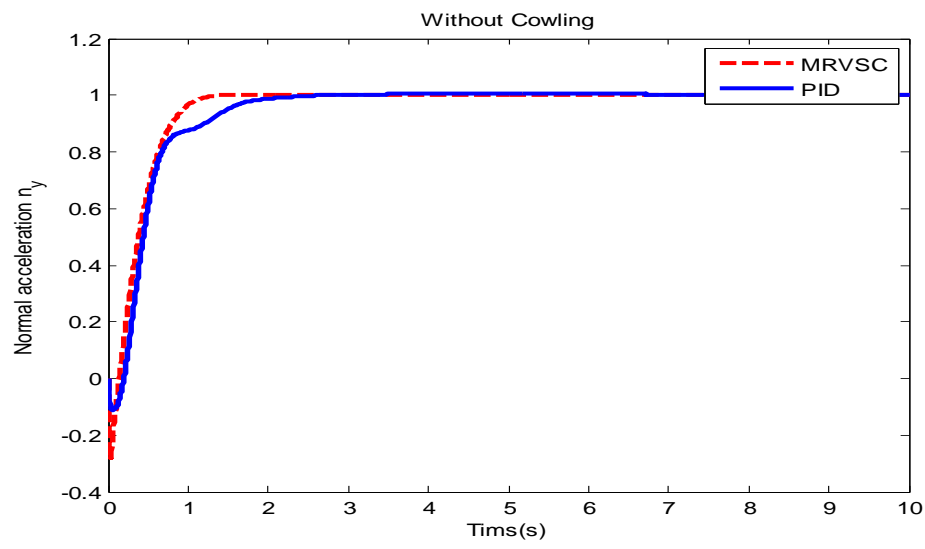

Fig. 3. Normal acceleration step response without cowling.

\section{MRVSC Design and Simulation of Roll Channel}

\subsection{MRVSC Design of Roll Channel}

The design procedures of roll channel are the same as pitch channel and no more explanation is 
presented here. The state variable of reference model is $X_{m}=\left[\begin{array}{ll}\gamma_{m} & \omega_{x m}\end{array}\right]^{T}$, and the state equation of reference model can be described as:

$$
\left[\begin{array}{l}
\dot{\gamma}_{m} \\
\dot{\omega}_{x m}
\end{array}\right]=\left[\begin{array}{cc}
0 & 1 \\
-\frac{1}{T_{m}^{2}} & -\frac{2 \xi_{m}}{T_{m}}
\end{array}\right]\left[\begin{array}{l}
\gamma_{m} \\
\omega_{x m}
\end{array}\right]+\left[\begin{array}{c}
0 \\
\frac{1}{T_{m}^{2}}
\end{array}\right] \gamma_{c}
$$

The reference model control law can be written as follow:

$$
u_{m r}=\frac{1}{c_{3} T_{m}^{2}}\left(\gamma-\gamma_{c}\right)+\frac{2 \xi_{m}-T_{m} c_{1}}{c_{3} T_{m}} \omega_{x}
$$

And the variable structure control law $u_{v s}$ is shown in the following equation:

$$
u_{v s}=\frac{g(t)}{-c_{3}} \operatorname{sgn}(S)
$$

\subsection{Performance Check of the Main Design Point of Roll Channel}

Variable structure control scheme of hypersonic vehicle roll channel is roll angle + roll rate control, and the schematics of control system is shown as Fig. 4.

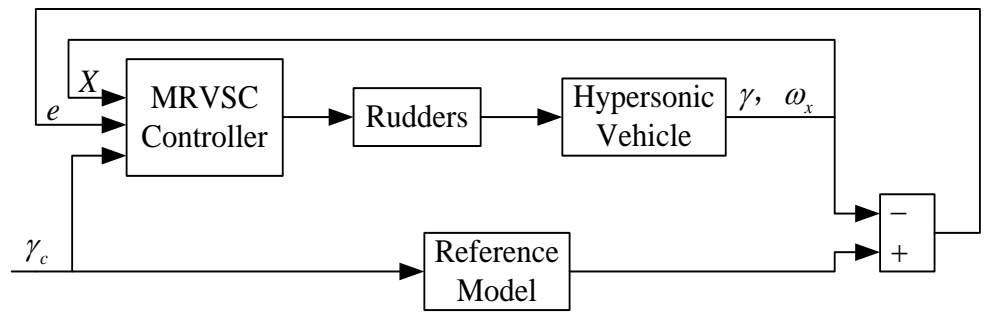

Fig. 4. Control system of roll channel.

Simulation conditions are the same as mentioned above. Time-domain characteristics of main design point are shown in Fig. 5 and Fig. 6.

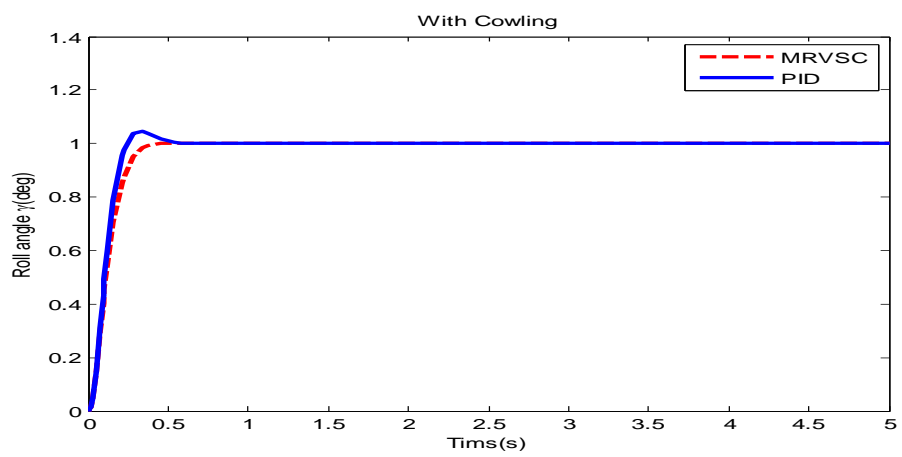

Fig. 5. Roll angle step response with cowling.

\section{Six Degree of Freedom Simulation and Analysis}

To validate the performance of MRVSC, examine system dynamic quality and steady performance of the 
three-channel control systems, 6-DOF simulation results of a STT hypersonic vehicle are shown below.

Simulation conditions: initial position is $X_{0}=0 \mathrm{~m}, Y_{0}=26000 \mathrm{~m}, Z_{0}=0 \mathrm{~m}$; initial velocity is $V_{x 0}=6 M a$, $V_{y 0}=0 \mathrm{~m} / \mathrm{s}, V_{z 0}=0 \mathrm{~m} / \mathrm{s}$. Time constant of the actuator is $0.008 \mathrm{~s}$, and damping coefficient is 0.6. Time constant of the rate gyro $0.002 \mathrm{~s}$, and damping coefficient is 0.7 . Lateral maneuver will be performed at the time of $50 \mathrm{~s}$ and $60 \mathrm{~s}$ for $3 \mathrm{~s}$, and the magnitude is 0.5. The simulation results are shown in Fig. 7 and Fig. 8.

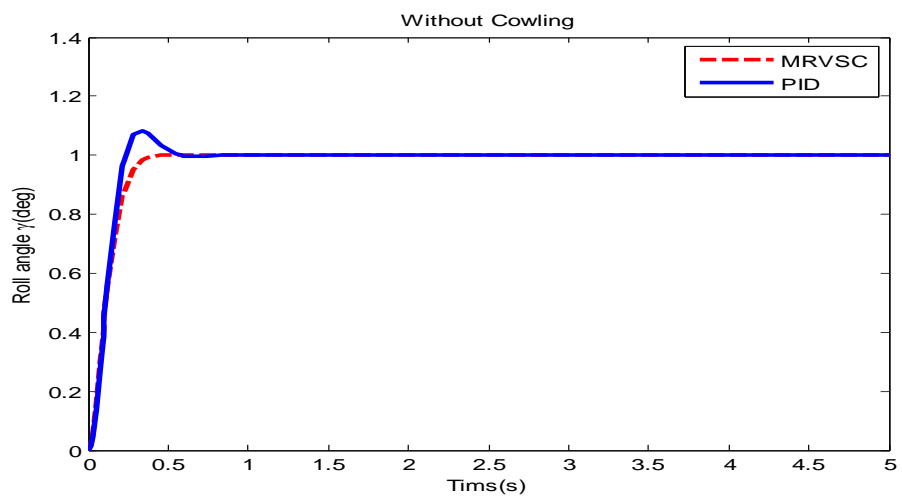

Fig. 6. Roll angle step response without cowling.

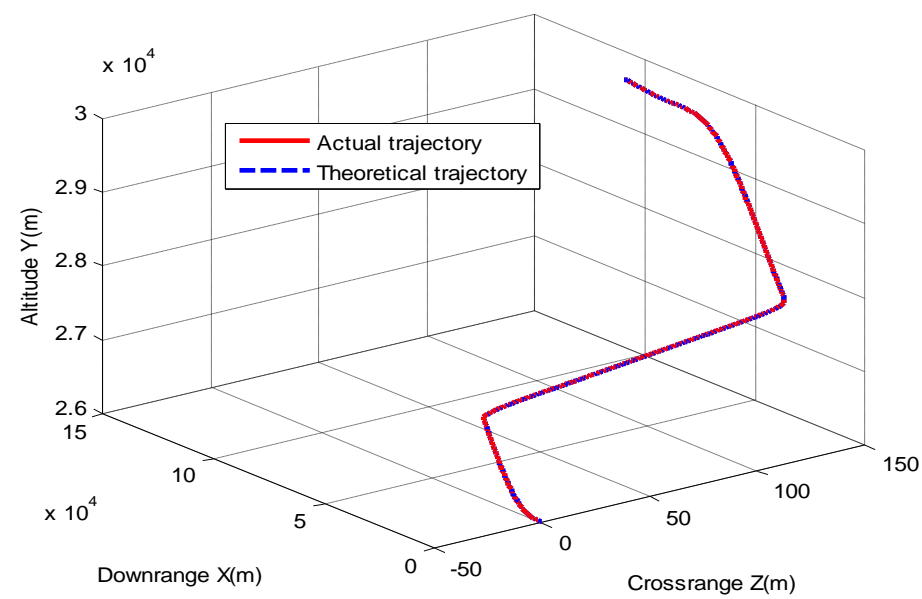

Fig. 7. Curve of trajectory tracking.

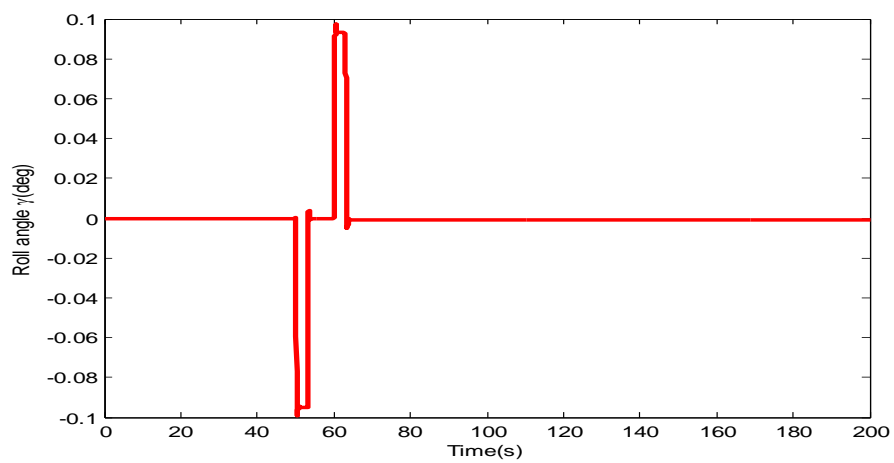

Fig. 8. Response curve of roll angle.

Though Fig. 7 and Fig. 8, we can find that, MRVSC can effectively control the STT hypersonic vehicle track the ideal trajectory, and almost keep roll angle unchanged. It means that MRVSC enables to control the system enter the sliding mode in a short time. And in case of dramatic changes in altitude, it can overcome 
parameter perturbation easily, and show good dynamic property and strong robustness.

\section{Conclusion}

We use the MRVSC scheme to design the model reference variable structural controller for the pitch and roll channel of a hypersonic vehicle. The scheme utilizes the adaptiveness of model reference and the merits of variable structural control. We compare the performances of main design points of each channel. The comparison results show that MRVSC can keep the control effects unchangeable under the circumstance of changeable parameters of the control system. But under the circumstance of parametric variation, PID controller has poorer control effects; the control system needs more control time or has larger overshooting. This shows that under the circumstance of parametric variation, MRVSC can ensure the robustness of a control system and its design by overcoming the shortcomings of the conventional PID controller such as uncertainties and poor adaptiveness when interference exceeds a certain limit. Finally, we give the overall-ballistic 6-DOF simulation results, which further verify that MRVSC has strong robustness to uncertainties.

\section{References}

[1] Zhu, Y. M. (2009, June). Nonlinear Control for a Hypersonic Flight Vehicle. Northeastern University.

[2] Li, K. F., \& Ren, Z. (2006). Study on robust variable structure control for hypersonic missile in reentry. Control Technology of Tactical Missile, 54(3), 1-4.

[3] Man, Z. H., Paplinski, A. P., \& Wu, H. R. (1994). A robust MIMO terminal sliding mode control scheme for rigid robot manipulators. IEEE Transaction on Automatic Control, 39, 2464-2469.

[4] Yu, X. H., et al. (2004). Fast terminal sliding mode control design for nonlinear dynamical systems. IEEE Transactions on Circuits and Systems-I: Fundamental Theory and Applications, 49(2), 261-265.

[5] Yu, S. H., \& Yu, X. H. (2000, December). Robust global terminal sliding mode control of SISO linear uncertain systems. Proceedings of the 35th Conference on Decision and Control, Sydney, Australia.

[6] Zhou, F. Q., Wang, Y., Zhou, J., \& Guo, J. G. (2011, January). Design of variable structure controller for hypersonic coupled vehicle system. Journal of Astronautics, 32(1), 66-71.

[7] Xiong, K., Xia, Z. X., \& Gun, Z. Y. (2012, February). Decoupling controller design for bank-to-turn hypersonic vehicle based on sliding mode variable structure. Flight Dynamics, 30(1), 61-65.

[8] Gao, H. Y., \& Cai, Y. L. (2014, January). Sliding mode predictive control for hypersonic vehicle. Journal of Xi'an Jiaotong University, 48(1), 67-72.

[9] Gao, D. X., Sun, Z. Q., \& Du, T. R. (2007). Dynamic surface control for hypersonic aircraft using fuzzy logic system. Proceedings of International Conference on Automation and Logistics (pp. 2314-2319).

[10] Li, Y. J., \& Zhang, K. (2005). Adaptive Control Theory and Application (1st ed.). Xi'an: Northwestern Polytechnical University.

[11] Liu, T., Yan, B. B., Gu, W. J., Yu, \& Y. F., (2014, August). Sliding mode variable structure control law design for hypersonic vehicle. Flight Dynamics, 32(4), 325-328.

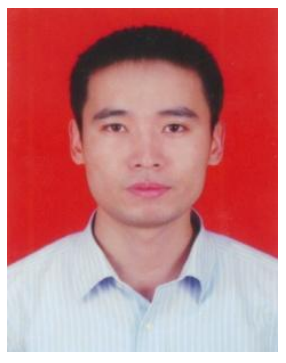

Jianli Wei was born in Datong Shanxi Province of P.R. China in 1978. Then he received the bachelor of engineering degree in aircraft design from Northwestern Polytechnical University, Xi'an Shaanxi, P.R. China, in 2002, and the master of engineering and Ph.D. degrees in navigation, guidance and control technology from Northwestern Polytechnical University, Xi'an Shaanxi, P.R. China, in 2005 and 2011, respectively.

He became a teacher of College of Astronautics of Northwestern Polytechnical University after he got his Ph.D. There, he published a number of articles and books with 
his colleagues, such as Research of composite rudder control of hypersonic vehicle, Shenyang Liaoning, P.R. China, in 2013; Square-root divided difference rauch-tung-striebel smoother, Harbin Heilongjiang, P.R. China, in 2012; Precision guided missile guidance control system simulation, Xi'an Shaanxi, P.R. China, in 2010; and so forth. His main direction of studying currently is control technology of hypersonic vehicle.

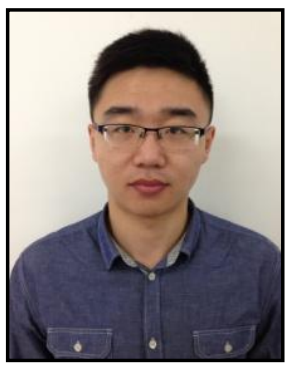

Shida Tian was born in Rizhao Shandong Province of P.R. China in 1991. In 2013, he got a bachelor of engineering degree from Northwestern Polytechnical University, Xi'an, China. Now he is a master in reading of Northwestern Polytechnical University, and his main direction of studying currently is guidance and control of aircraft.

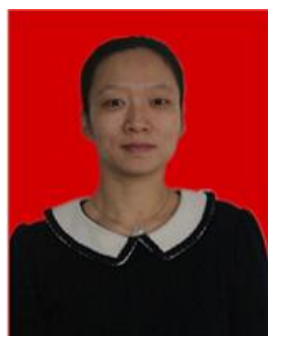

Xinghua Yao was born in Baoji Shaanxi Province of P.R. China in 1979. She became a teacher of Ming De College, Northwestern Polytechnical University in 2005, Her main direction of studying currently is computer control and simulation. 